\title{
Horse-Like Walking, Trotting and Galloping derived from Kinematic Motion Primitives (kMPs) and their Application to Walk/Trot Transitions in a Compliant Quadruped Robot
}

\author{
Federico L. Moro - Alexander Spröwitz • Alexandre Tuleu • \\ Massimo Vespignani · Nikos G. Tsagarakis · \\ Auke J. Ijspeert · Darwin G. Caldwell
}

Received: date / Accepted: date

\begin{abstract}
This manuscript proposes a method to directly transfer the features of horse walking, trotting, and galloping to a quadruped robot, with the aim of creating a much more natural (horse-like) locomotion profile. A Principal Component Analysis (PCA) on horse joint trajectories shows that walk, trot, and gallop can be described by a set of four kinematic Motion Primitives (kMPs). These kMPs are used to generate valid, stable gaits that are tested on a compliant quadruped robot. Tests on the effects of gait frequency scaling follow: results indicate a speed-optimal walking frequency around $3.4 \mathrm{~Hz}$, and an optimal trotting frequency around $4 \mathrm{~Hz}$. Following, a criterion to synthesize gait transitions is proposed, and the walk/trot transitions are successfully tested on the robot. The performance of the robot when the transitions are scaled in frequency is evaluated by means of roll and pitch angle phase plots.
\end{abstract}

Keywords Quadrupedal locomotion, kinematic Motion Primitives (kMPs), horse-like gaits, gait transitions, compliant quadruped robot

Federico L. Moro, Nikos G. Tsagarakis, Darwin G. Caldwell Department of Advanced Robotics

Istituto Italiano di Tecnologia (IIT)

Via Morego 30, 16163 Genova, Italy

Alexander Spröwitz, Alexandre Tuleu, Massimo Vespignani, Auke J. Ijspeert

Biorobotics Laboratory

École Polytechnique Fédérale de Lausanne (EPFL)

Station 14, 1015 Lausanne, Switzerland

Corresponding author:

Federico L. Moro

Tel.: +3901071781 481

Fax: +3901071781232

E-mail: federico.moro@iit.it

\section{Introduction}

In most scenarios where the terrain is smooth (roads) or comparatively smooth (open fields) wheeled vehicles, often in the form of four wheel drive, can cope extremely well. However, where the landscape is extremely steep, littered with rocks, boulders, etc., either occurring naturally or as rubble after some form of devastation (e.g., earthquake, war), then wheeled vehicles can have difficulties operating, particularly if there is a need for complex maneuvering in relatively confined space. Under such circumstances legged systems may have distinct operational advantages, due to the versatility and flexibility they provide, and the resultant ability to adapt much more easily to complex environments within irregular terrain (Raibert et al, 2008). For this reason, interest in legged robots has steadily grown within the robotics community in the past two decades.

Quadrupeds, in particular, are more stable and typically more powerful than bipeds, and these characteristics can make them well suited for outdoor tasks, such as assisting humans transporting heavy loads for them, or even operating autonomously in a hostile environment. To try to exploit the potential of legged locomotion a number of solutions have been developed for robots of varying size, powered by diverse energy sources. Among these, notable examples include Fujita and Kitano (1998); Berns et al (1998); Canderle and Caldwell (2000); Nichol et al (2004); Poulakakis et al (2005); Buehler et al (2005); Zhang et al (2005); Kimura et al (2007); Raibert et al (2008); Hirose et al (2009); Semini et al (2011).

Quadrupedal locomotion presents good intrinsic stability features, but the coordination of the motion of four legs is non-trivial to control. Different trajectory generation techniques have been adopted (Kimura et al, 
1989; Raibert, 1990; Sakakibara et al, 1990; Kramy and Orin, 2003; Golubovic and Hu, 2003a,b; Tsujita et al, 2005; $\mathrm{Hu}$ and $\mathrm{Gu}, 2005$; Iida et al, 2005; Kim et al, 2006; Rebula et al, 2007; Pongas et al, 2007; Hebbel et al, 2007; Chae and Park, 2008; Kim et al, 2008), and the efforts in this field of research led to proficient results in the development of different gaits, from the slow walk to gallop. Raibert (1986) provides a good overview of legged locomotion, with particular attention given to quadrupeds.

The results achieved, however, are still far from the proficient, efficient, and fluid motion of animal locomotion. For this reason many researchers have sought to analyze animal gait (Hoyt and Taylor, 1981), with the aim of reproducing this on robots. Different methods for biological inspirations have been proposed: Collins and Richmond (1994); Fukuoka et al (1999, 2003); Billard and Ijspeert (2000); Fujii et al (2002); Witte et al (2003); Ishii et al (2004); Son et al (2006); Cappelletto et al (2006, 2007); Righetti and Ijspeert (2008); Rutishauser et al (2008); Liu et al (2009), with the well known theory of Central Pattern Generators (CPG) forming a common theme. According to the theory of CPG, open-loop signals are produced in the spinal cord and sent to the limbs to produce the motion, reducing significantly the complexity of controlling a vast variety of motions. Sensor information is then used to correct the error and adapt the motion to the peculiar situation. Ijspeert (2008) and Dégallier Rochat and Ijspeert (2010) provide a comprehensive overview on the biologically inspired methods, particularly CPG-based, for robot locomotion. The effects of CPG can be noticed at different levels in the form of Primitives: at the level of muscles, with EMGs, or at the level of the kinematics, from the joint trajectories. The Primitives are basic elements (signals, stimuli, etc.) underlying human/animal motion, and can be used as open-loop modules to generate motion for robots (Degallier et al, 2008). Moro et al $(2011,2012 \mathrm{a}, \mathrm{b})$ proposed a method to extract the kinematic Motion Primitives ( $k M P s$ ) from human data (joint angle trajectories). A set of kMPs, defined as "invariant waveforms [...] sufficient to explain a wide variety of complex coordinated motions" (Moro et al, $2012 \mathrm{~b}$, page 1 ), is identified, and then used to generate a human-like walking for the biped robot COMAN.

The research described in this paper aimed to identify the kMPs of horse locomotion, and use them to directly transfer the biological features of horse locomotion to a quadruped robot. In Section 2 the kMPs extracted from walking, trotting, and galloping are analyzed. The compliant quadruped robot (Spröwitz et al, 2012) that was used to test the horse-like trajectories synthesized is introduced in Section 3. Next, in Section 4, the method

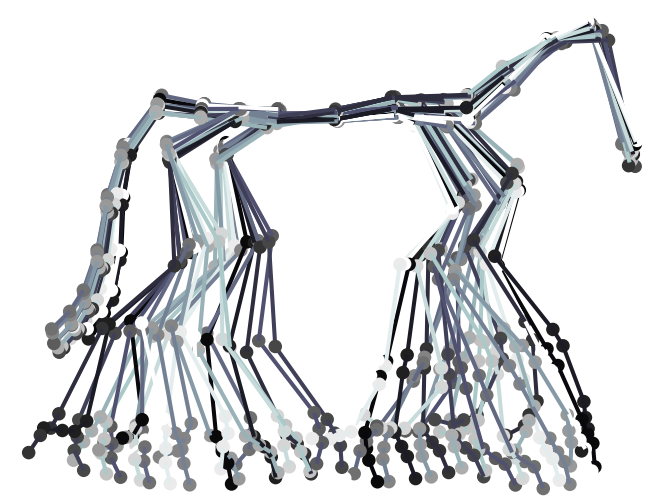

(a) The horse walking on a treadmill

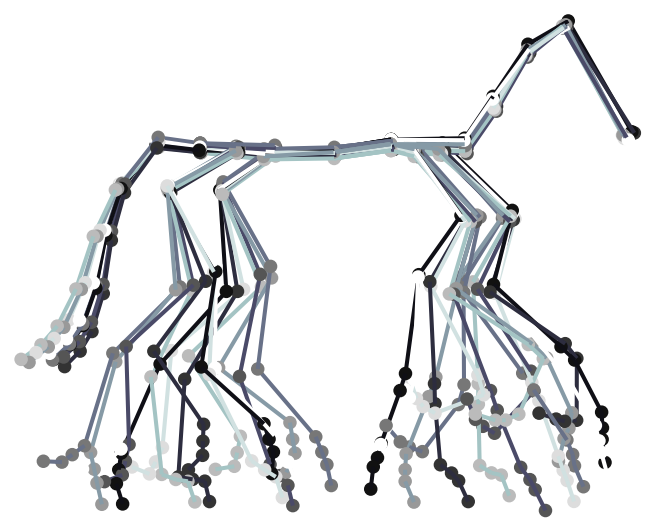

(b) The horse trotting on a treadmill

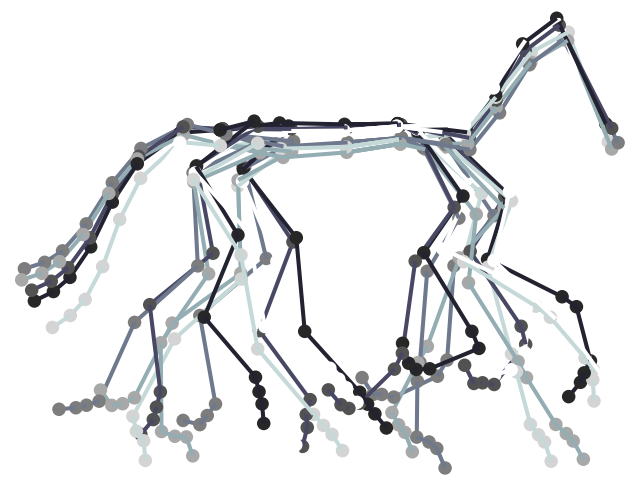

(c) The horse galloping on a treadmill

Fig. 1 The source data (visualization at $12 \mathrm{~Hz}$ ) of the horse a) walking, b) trotting, and c) galloping on a treadmill. 
used to generate these trajectories by reconstruction from kMPs is presented. Subsequently, the trajectories were scaled in frequency to evaluate the performance of locomotion at different gait frequencies. This analysis was triggered by the research presented in Heglund and Taylor (1988). Finally, Section 5 proposes a gait transition strategy. Developing an effective gait transition is fundamental for achieving a good performance in quadrupedal locomotion. Vilensky et al (1991); Inagaki and Kobayashi (1993); Lin and Song (2002); Griffin et al (2004) proposed interesting research on this topic. Walk to trot and trot to walk transitions generated according to the proposed strategy were successfully tested on the quadruped robot.

\section{Extraction of the horse kinematic Motion Primitives (kMPs)}

The source data used in these experiments were purchased from the U.K. based commercial company Kinetic Impulse. These data consist of the joint trajectories of a horse (Figure 1) performing a walking gait, a trotting gait, and a galloping gait on a treadmill (horse dimensions: hips to the ground at rest position: $1.472 \mathrm{~m}$; hips to shoulders distance: $1.197 \mathrm{~m}$; right hip to left hip distance: $0.269 \mathrm{~m}$; right shoulder to left shoulder distance: $0.205 \mathrm{~m}$. These last two data refer to the distance between joints that are internal to the horse body, and are the first joints of the limbs from the spine). For each of the three gaits, three sequential gait cycles were arbitrarily selected, and the respective full-body joint angle trajectories (only those with a range of motion of at least $10^{\circ}$ were considered) were averaged to reduce the peculiarity of the single cycle.

On these trajectories a Principal Component Analysis (PCA) was subsequently applied. For each of the three gaits the first four components were selected. In the case of the walk gait these components together accounted for $97 \%$ of the variance. Similar values were observed also in the case of the trot gait, and in the case of the gallop gait, with a cumulative percentage of variance accounted for $96 \%$ and $97 \%$, respectively. These four components, normalized in time (from $0 \%$ to $100 \%$ of gait cycle) and amplitude (such that the maximum absolute value was 1) form the four kinematic Motion Primitives (kMPs) of walking, trotting, and galloping (Figure 2). Further details on the extraction of the kinematic Motion Primitives (kMPs) can be found in Moro et al (2011, 2012a,b).
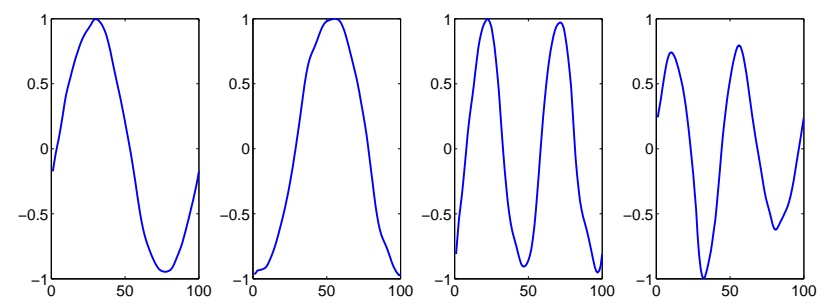

(a) The four kMPs extracted from walk
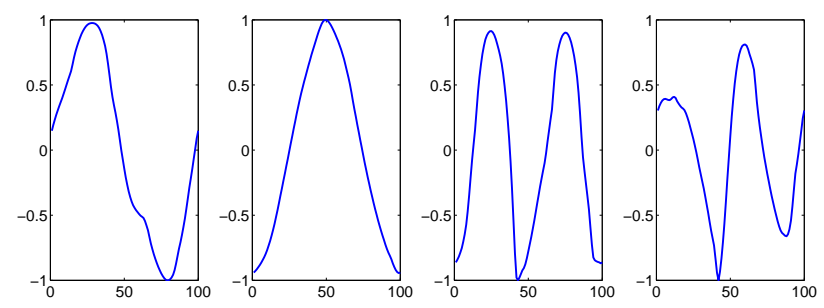

(b) The four kMPs extracted from trot
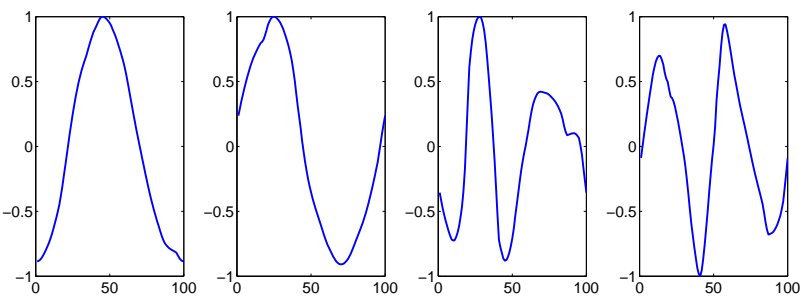

(c) The four kMPs extracted from gallop

Fig. 2 The kinematic Motion Primitives

\subsection{Comparison of the different gaits and statistical analysis of similarity}

A similarity among the kMPs extracted from the three gaits can be already noticed by observation of Figure 2 . If in the walk and the trot gaits the order of the kMPs remains the same, the first and the second kMPs of gallop have an inverted order with respect to the other two gaits. The third and the fourth kMPs, instead, maintain the same order in all the gaits analyzed. In Figure 3 the corresponding four kMPs from the walk, trot, and gallop gaits are superimposed to facilitate a comparison.

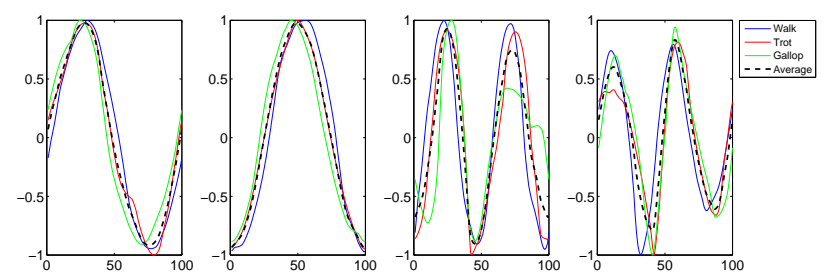

Fig. 3 Comparison among the corresponding kMPs extracted from the walk, trot, and gallop gaits

The similarity in terms of shape and phase observed by visualization in Figure 3 was confirmed by means of a 
statistical analysis that provided a quantification of the degree of similarity between gaits. To quantify the similarity between two sets of kMPs the maximum crosscovariance between each corresponding kMP (sliding in time) was calculated, and normalized so that the autocovariance is 1 . The delay between any two compared kMPs is also returned. This value indicates how much time-slip is needed in a signal to maximize the crosscovariance. Again this is normalized so that a slip of an entire cycle has a value of 1 . An indication of the similarity (and delay) between the two entire sets is provided as the weighted average of the cross-covariance of the different kMPs. The weights used are the average of the corresponding variance explained by the kMPs compared. More details on the methodology adopted to derive the degree of similarity between two gaits can be found in Moro et al (2012b). The following tables summarize the results of the analysis:

Table 1 Statistical analysis of similarity among kMPs extracted from walk $(\mathrm{W})$, trot $(\mathrm{T})$, and gallop $(\mathrm{G})$ - Crosscovariance

\begin{tabular}{|c||c|c|c|c||c||c|}
\hline & 1st & 2nd & 3rd & 4th & Average & Weighted \\
\hline \hline W_T & 0.9844 & 0.9783 & 0.9207 & 0.9141 & 0.9494 & 0.9722 \\
\hline T_G & 0.9934 & 0.9170 & 0.7282 & 0.9674 & 0.9015 & 0.9277 \\
\hline W_G & 0.9876 & 0.9713 & 0.8120 & 0.9418 & 0.9282 & 0.9560 \\
\hline
\end{tabular}

Table 2 Statistical analysis of similarity among kMPs extracted from walk $(\mathrm{W})$, trot $(\mathrm{T})$, and gallop $(\mathrm{G})$ - Delay

\begin{tabular}{|c||c|c|c|c||c||c|}
\hline & 1st & 2nd & 3rd & 4th & Average & Weighted \\
\hline \hline W_T & 0.03 & 0 & 0.03 & -0.04 & 0.005 & 0.0227 \\
\hline T_G & 0.03 & 0 & -0.01 & -0.01 & 0.0025 & 0.0144 \\
\hline W_G & 0.06 & -0.02 & -0.06 & -0.06 & -0.02 & 0.0482 \\
\hline
\end{tabular}

The kMPs of the three gaits have a similarity that is in all cases significant (Table 1). This led to an overall similarity between walk and trot gait of $97 \%$. This value reduced to $93 \%$ in the case of trot and gallop. The last comparison, between walk and gallop, have a degree of similarity of $96 \%$. In Table 2 the delay between kMPs is reported: it can be noticed that the weighted average delay is smaller than $5 \%$ in all three comparisons between gaits. This result indicates an evident correlation between the kMPs of the different gaits. A possible interpretation is that the $\mathrm{kMPs}$ extracted from walk, trot, and gallop are in fact the same set of $\mathrm{kMPs}$, that together are sufficient to describe the three different gaits. In the next section the inverse process to reconstruct the joint trajectories from this set of kMPs will be presented.

\subsection{Trajectories reconstruction}

The analysis reported in the previous section shows that a small set of kMPs can describe the kinematics of different horse gaits. The following formula describes how to reconstruct the joint trajectories from the kMPs identified:

$$
\left[\begin{array}{c}
q_{1} \\
\vdots \\
q_{i}
\end{array}\right]=\left[\begin{array}{ccc}
s_{1,1} & \ldots & s_{1, j} \\
\vdots & \ddots & \vdots \\
s_{i, 1} & \ldots & s_{i, j}
\end{array}\right] \times\left[\begin{array}{c}
P_{1} \\
\vdots \\
P_{j}
\end{array}\right]+\left[\begin{array}{c}
\overline{Z_{1}} \\
\vdots \\
\overline{Z_{i}}
\end{array}\right]
$$

where $\left[q_{1} \ldots q_{i}\right] \in \mathbb{R}^{i}$ represents the vector of the joint trajectories, $\left[P_{1} \ldots P_{j}\right] \in \mathbb{R}^{j}$ is the vector of the kinematic Motion Primitives (kMPs), $\left[\overline{Z_{1}} \ldots \overline{Z_{i}}\right] \in \mathbb{R}^{i}$ is a zero offset mean vector. $\overline{Z_{i}}$ is added back to the $i^{\text {th }}$ joint trajectory (PCA was applied on the zero-mean normalized trajectories). The matrix $\left[s_{1,1} \ldots s_{i, j}\right] \in \mathbb{R}^{i, j}$ represents the motion primitives synergy map. The joint trajectories are therefore a linear combination of the kMPs through the motion primitives synergetic coefficients in matrix S. The columns of this matrix map the contribution of each primitive to the joint space.

Since horse walk, trot, and gallop can be represented by the same 4 components, it is possible to reconstruct different gaits from the same $\mathrm{kMPs}$, reproducing the features of the horse gait, by means of a proper selection of the values of the matrix S. The choice of these values, however, is not trivial. In Section 4 the method adopted to reconstruct horse-like trajectories for a compliant quadruped robot (presented in the next section) will be reported.

\section{The experimental compliant quadruped robot}

The quadruped robot used for experimentation (Figure 4) was designed at the Biorobotics laboratory at EPFL, Switzerland. Its leg design was roughly based on the pantograph leg design proposed by Witte et al (2003). A three-segmented, panthograph mechanism was implemented for both forelimbs and hind limbs. The leg mechanism is a passively spring loaded (diagonal spring) and gravity compensating mechanism with parallel cable actuation. The parallel spring (Figure 4) enables the robot's lower leg segment to leave the parallel orientation relative to the leg's upper segment, if an external force is present. Each leg is actuated by two RC servo motors. Motor axes were mounted perpendicular to the parasaggital robot plane. The hip (hind) or (shoulder) RC servo motors are protracting (swing forward) and retracting (swing backward) the leg. RC 


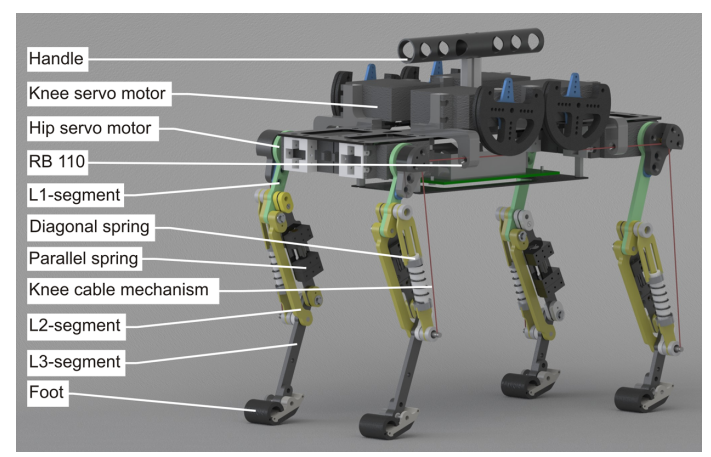

Fig. 4 The compliant quadruped robot used for experimentation (rendered image), developed at Biorobotics laboratory, EPFL, Switzerland (Spröwitz et al, 2012). Snapshots of the real robot during walking and trotting are provided in Fig. 9. This robot configuration weights $m \approx 1.1 \mathrm{~kg}$, and has the size of a small house cat. Power to the robot was provided through a tether. During experiments, the tether was carefully kept slack.

Table 3 Robot characteristics and comparison with the horse dimensions. Distances for the horse present distances of the tracking markers, distances of functional joints might differ slightly.

\begin{tabular}{lcc} 
Characteristic & Value & Horse \\
\hline Mass $\mathrm{m}_{\text {robot }}$ & $1.1 \mathrm{~kg}$ & \\
Standing hip height $\mathrm{h}_{\text {hip }}$ & $0.158 \mathrm{~m}$ & $1.472 \mathrm{~m}$ \\
Distance $\mathrm{d}_{\text {sh }-\mathrm{sh}}$ & $0.1 \mathrm{~m}$ & $0.205 \mathrm{~m}$ \\
Distance $\mathrm{d}_{\text {hip }}$ hip & $0.1 \mathrm{~m}$ & $0.269 \mathrm{~m}$ \\
Distance $\mathrm{d}_{\text {hip }}-\mathrm{sh}$ & $0.205 \mathrm{~m}$ & $1.197 \mathrm{~m}$ \\
RC servo motors $(8 \mathrm{x})$ & Kondo Krs 2350 ics & \\
Control board & Roboard RB110 & \\
Operation system & Linux Xenomai & \\
Communication & Wifi Via VT6655 & \\
Power supply (tethered) & $10.5 \mathrm{~V}$ & \\
Stall torque RC servo & $2 \mathrm{Nm}(6 \mathrm{~V})$ & \\
Speed max RC servo & $0.16^{\circ} / 60^{\circ}(6 \mathrm{~V})$ & \\
Spr. stiffness $\mathrm{k}_{\text {diagonal }}$ & $2300 \mathrm{~N} / \mathrm{m}$ & \\
Spr. stiffness $k_{\text {paral,front }}$ & $4800 \mathrm{~N} / \mathrm{m}$ & \\
&
\end{tabular}

servo motors at the knee provide flexion-extension motion. Both motors are mounted directly at the trunk, this reduces moving leg masses. The cable mechanism is also acting as an automatic clutch, for external forces applying a flexing torque to the leg. Robot feet (length $14 \mathrm{~mm}$ ) are mounted at the distal end of the leg. Instead of choosing simpler cylinder shaped surfaces, the robot's feet are separate segments, mounted at the L3segment by small torsion springs. During earlier experiments we found this construction was more robust against sliding, at e.g. different ground surfaces and more independent from the foot material used. Feet were covered with standard Duct tape in all experiments. The system specifications are show in Table 3.

To calculate the leg's inverse kinematics (IK) we neglect the effect of the in-series leg spring element ("parallel spring"), which only acts when leg-external force are applied. With this simplification, the inverse kine-

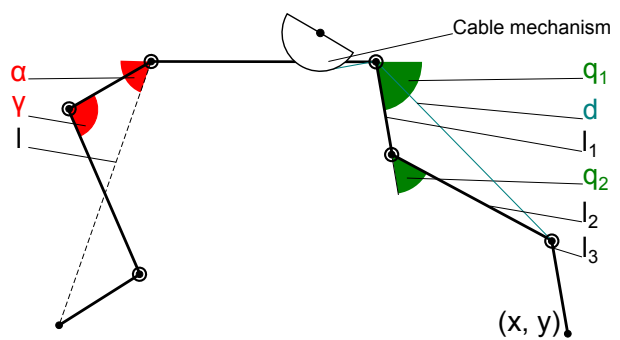

Fig. 5 Schematic presentation of the pantograph leg mechanism, forward is to the left. Leg parameters $\left(l_{1}, l_{2}, l_{3}, l\right)$ are given, as well as IK control parameters $\left(q_{1}, q_{2}, d\right)$. Task space is presented as the heel point $((x, y))$, and $\alpha$ as the leg angle. The half-disc presents the knee RC servo motor.

matics mechanics of the pantograph mechanism can be reduced to that of a two-link mechanism. Leg-IK are provided in Equations 2-5, and a schematic presentation with leg parameters is given in Fig. 5 .

$$
\begin{aligned}
q_{2} & =\arccos \frac{x^{2}+y^{2}-\left(l_{1}+l_{3}\right)^{2}-l_{2}^{2}}{2\left(l_{1}+l_{3}\right) l_{2}} \\
\alpha & =\arctan 2\left(\frac{y}{x}\right) \\
q_{1} & =\arcsin \left(\frac{l_{2} \sin q_{2}}{\sqrt{x^{2}+y^{2}}}\right)+\arctan 2\left(\frac{y}{x}\right)
\end{aligned}
$$

The cable mechanism is acting on distance $d$, not directly on joint angle $q_{2} . d$ is calculated through:

$d=\sqrt{l_{1}^{2}+l_{2}^{2}+2 l_{1} l_{2} \cos q_{2}}$

\section{Walking, trotting, and galloping with a compliant quadruped robot}

The procedure to generate joint trajectories by reconstruction from kMPs was presented in Section 2.2. As anticipated, however, the values of matrix $\mathrm{S}$ (in Equation 1) are generally not known. In the next section the method adopted to overcome this issue and reconstruct a valid gait for a quadruped robot from kMPs is described. Results applying joint trajectories and experiments with walk, trot and gallop gaits at the quadruped robot are reported in Section 4.3. Stability is not directly being controlled. The proposed method deals exclusively with kinematic quantities. The gaits generated, though, were observed to be stable, i.e., the robot could successfully perform the tested gaits without falling.

\subsection{Reconstruction of the foot trajectories}

The similarity between kMPs of different horse gaits, observed in the comparative analysis described in Section 2.1, suggests that a unique set of four kMPs is at 
the basis of walk, trot, and gallop gaits. Hence, these kMPs can ideally be used to reconstruct any of the three gaits considered, just by varying the values of matrix $S$ (Equation 1).

Since the values required to reconstruct a specific gait are not known, however, it was not possible to follow this procedure. The kMPs extracted from each gait, instead, were used to reconstruct the corresponding gait for the quadruped robot. PCA was applied on an enriched set of trajectories, that includes not only the joint trajectories, but also the four Cartesian feet trajectories with respect to a frame located in the middle of the spine. These trajectories are coupled with the joint trajectories, and it was verified that the kMPs extracted do not change when this information is added. In this way, though, the coefficients to reconstruct the Cartesian feet trajectories of the horse are known. The reconstructed feet trajectories are then proportionally scaled down according to the dimensions of the robot. These trajectories had to be slightly modified to satisfy the mechanical constraints of the robot. They are first projected on the sagittal plane, since there is no adduction/abduction degree of freedom in the legs of the robot. Then their range of motion is further scaled down to ensure they do not exceed the joint limits. The range of motion of the legs of the robot, in fact, is smaller than it is for a horse, and this is mainly because the robot does not have a flexible spine. The reference positions for the motors of the robot are then derived from the foot trajectories with inverse kinematics, as described in Section 3. In our approach the differences between the kinematics of the horse and the kinematics of the robot are not a limitation. What is maintained is the end-effector (foot) shape. Our method do not require the robot to have the same kinematics as the horse. The robot could successfully perform a valid, stable locomotion in all three cases (Figure 9).

\subsubsection{Walk gait}

Figure 6 shows the foot trajectories for a walk gait reconstructed from kMPs. It is possible to notice the symmetry between left and right foot trajectories. The hindleg foot trajectories, in both cases, have the shape of a triangle with two rounded angles on the left and a spike on the right. The foreleg trajectories, instead, have a shape that reminds of a bean. The range of motion is also very similar. The gait frequency is maintained the same as the original one of the horse walking at $0.97 \mathrm{~Hz}$.
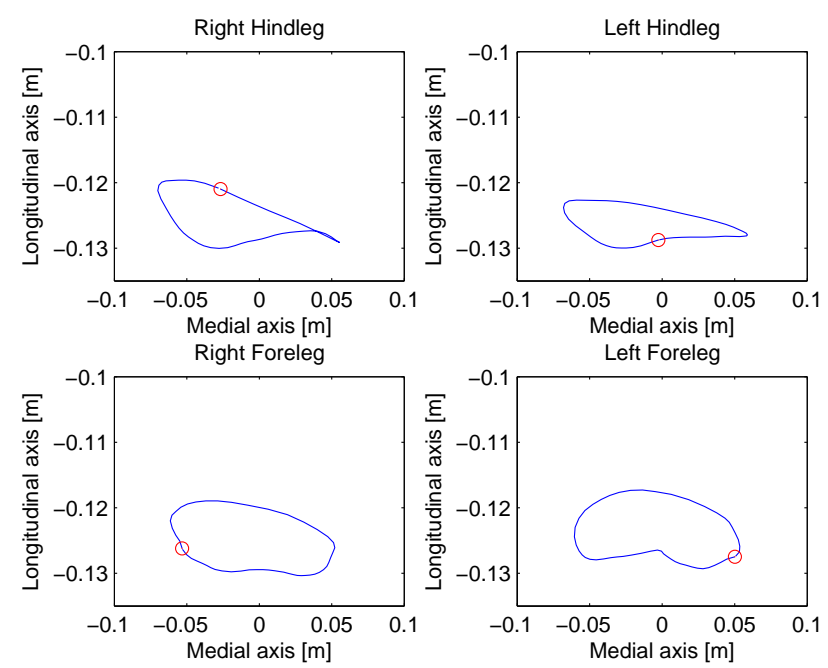

Fig. 6 The foot trajectories of the walk gait reconstructed from $\mathrm{kMPs}$

\subsubsection{Trot gait}

The foot trajectories for the trot gait reconstructed from kMPs are shown in Figure 7. The vertical displacement in the forelegs is wider than in the case of walking. The gait frequency is also higher at $1.59 \mathrm{~Hz}$, and is the same as that of the horse trotting.
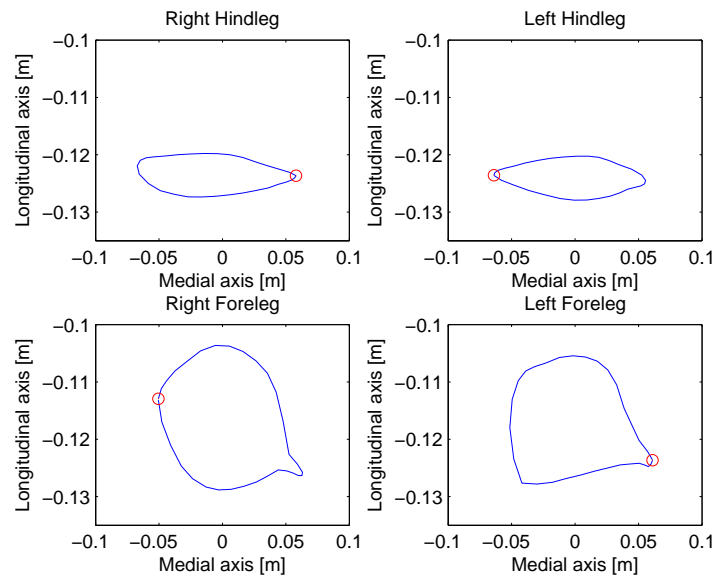

Fig. 7 The foot trajectories of the trot gait reconstructed from kMPs

\subsubsection{Gallop gait}

Figure 8 shows the foot trajectories of a gallop gait reconstructed from kMPs. The trajectories are less regular than in the other cases, and the displacement is wider in both directions. The gait frequency has increased further up to $2.01 \mathrm{~Hz}$, the same frequency of the horse galloping. 

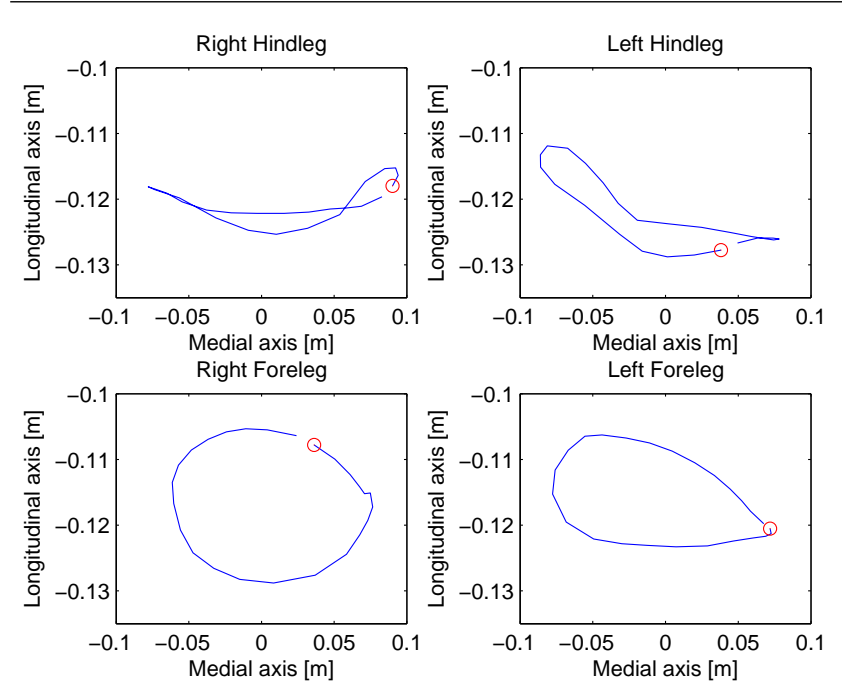

Fig. 8 The foot trajectories of the gallop gait reconstructed from kMPs

\subsection{Scaling the gaits in frequency}

The quadruped robot could successfully perform stable walk, trot, and gallop gaits at the original frequency. The performance of gallop though was not satisfactory. High speed video footage indicates that this was caused by the missing spine movement: while horses and other mammalian quadrupeds extensively use their spine at high speed gaits, the robot has a stiff trunk. The method proposed matches the kinematic features of both legs and spine motion of the horse with the legs motion of the robot. The resulting trajectories required high torques to be tracked, and this led to massive interlimb and contact forces that, consequently, caused major foot slippage. For this reason experiments described in this section focused on the walk and trot gaits.

In the gaits reconstructed from kMPs, the decision had been taken to maintain the same gait frequency as the original one. The dimensions of the robot, though, are different from those of the horse whose gait was used as source data. In the literature Heglund and Taylor (1988) reported on animal locomotion and body size scaling. They showed that gait frequency is inversely proportional to the dimensions of the animal itself. For this reason in following experiments gait frequencies of walk and trot gaits were increased systematically by a multiplication factor $m=[1: 0.5: 3.5]$. In case of trot gait, this resulted in locomotion frequencies between $f=1.6 \mathrm{~Hz}-5.6 \mathrm{~Hz}$. Heglund and Taylor (1988, page 306 , Figure $1 \mathrm{~F}$ ) reported that an animal of approximately equal size ( $\mathrm{dog}, m=0.96 \mathrm{~kg}$ ) as the quadruped robot reached its maximum trot-gait speed at a gait frequency of $f=4.5 \mathrm{~Hz}$. We expected a speed maximum roughly in the same frequency domain.

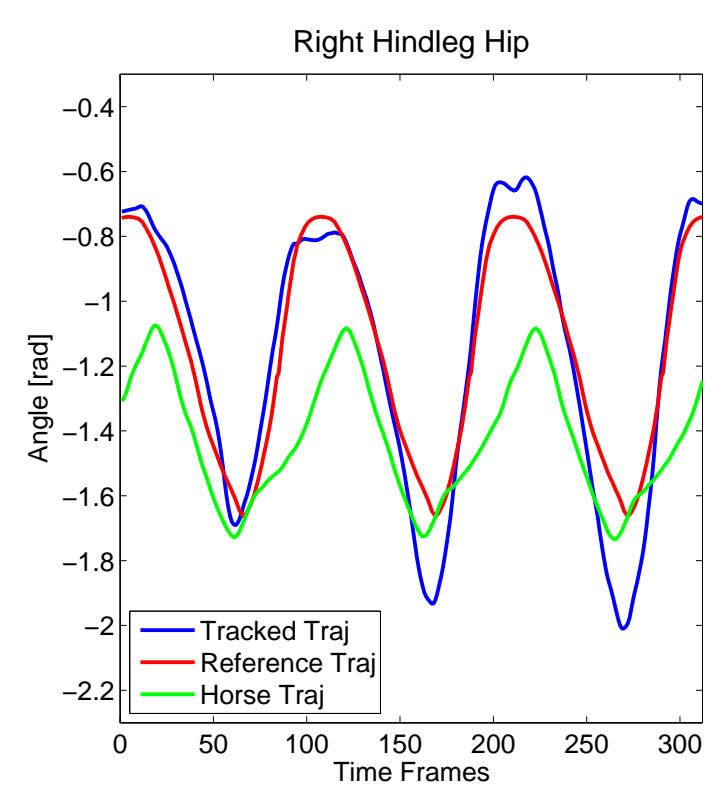

Fig. 10 The joint trajectory of the right hindleg hip (walk gait): comparison between horse and robot (reference and tracked) trajectories. It can be noticed that the robot trajectories have a wider displacement: this is because the robot does not have a flexible spine. The robot has to compensate the motion of the spine in the horse with the joints in its legs.

\subsection{Results for walk and trot gaits}

This section describes results from applying kMPs to the quadruped, compliant robot. We transferred walk, trot and gallop gait patterns to the robot, at their extracted base frequency. Base gait patterns were then scaled up in frequency, according to the robot dimensions with respect to the size of the horse (Section 4.2). Extracted kMPs were used to calculate servo trajectories, by applying inverse kinematics of the corresponding leg, front or hind. Control trajectories for proximal and distal joints were treated as position control signal, and sent as such to the position controlled RC servo motors. Figure 10 shows a comparison between the horse right hindleg hip joint trajectory, and the same joint for the robot (both reference and tracked trajectories are included) for the walk gait. As explained in Section 4.1 , the kinematics of the horse and that of the robot are significantly different, and this results in different joint trajectories to produce similar foot trajectories. In particular in can be noticed that the hip trajectory has a wider displacement robot than in the horse, and this is because the robot does not have a flexible spine. It has to compensate the motion of the spine in the horse with the joints in its legs.

The applied base frequencies for walk and trot were $f_{\text {walk }}=0.97 \mathrm{~Hz}$ and $f_{\text {trot }}=1.59 \mathrm{~Hz}$, respectively (Table 4). We linearly scaled the base frequencies with a 

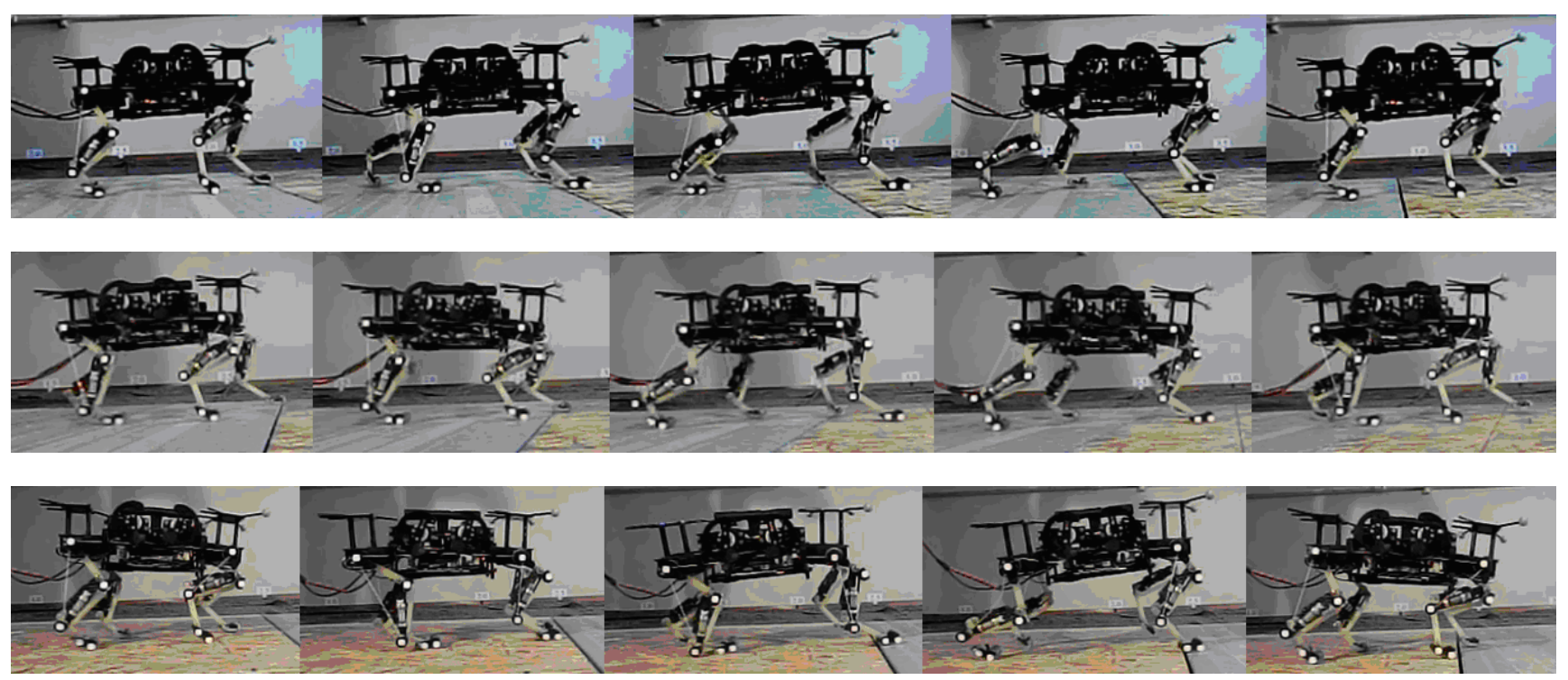

Fig. 9 Snapshots of walk (top), trot (center), and gallop (bottom) gait on the compliant quadruped robot.

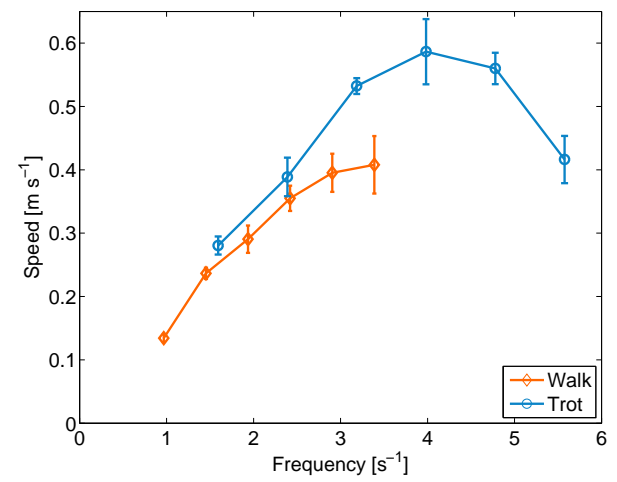

Fig. 11 Speed versus frequency of three different gaits, run on the compliant quadruped robot platform. Walking speed ranged from $0.14 \mathrm{~m} / \mathrm{s}$ to $0.42 \mathrm{~m} / \mathrm{s}$, with a speed-maximum at $f=2.9 \mathrm{~Hz}$. Trotting speed ranged from $0.29 \mathrm{~m} / \mathrm{s}$ to $0.62 \mathrm{~m} / \mathrm{s}$, with its speed maximum at $f=3.8 \mathrm{~Hz}$. For gallop, only the base-frequency of $f \approx 2 \mathrm{~Hz}$ lead to a stable gait, with a speed of $v=0.2 \mathrm{~m} / \mathrm{s}$.

multiplication factor $m=[1: 0.5: 3.5]$, and measured the resulting instantaneous robot speed for all the gaits.

Table 4 Cartesian speed of the robot walking, and trotting

\begin{tabular}{lrrlr}
$\mathrm{m}$ & $f_{\text {walk }}$ & $f_{\text {trot }}$ & $v_{\text {walk }}$ & $v_{\text {trot }}$ \\
\hline 1.0 & 0.97 & 1.59 & 0.13 & 0.28 \\
1.5 & 1.45 & 2.39 & 0.24 & 0.39 \\
2.0 & 1.94 & 3.18 & 0.29 & 0.53 \\
2.5 & 2.42 & 3.98 & 0.36 & 0.59 \\
3.0 & 2.90 & 4.77 & 0.40 & 0.56 \\
3.5 & 3.39 & 5.57 & 0.41 & 0.42
\end{tabular}

Walk gait speed Results in Figure 11 indicate that speed of the robot scaled almost linearly, with the base frequency being scaled up to a locomotion frequency of $f=2.42 \mathrm{~Hz}$. The average robot speed at $f=2.42 \mathrm{~Hz}$ was $v=0.35 \mathrm{~m} / \mathrm{s}$. For two higher frequencies $(2.9 \mathrm{~Hz}$ and $3.39 \mathrm{~Hz}$ ), robot speed still increased. Plots in Figure 11 indicate that the maximum walking speed was reached close to the highest applied locomotion frequency of $f=3.39 \mathrm{~Hz}$, with $v=0.43 \mathrm{~m} / \mathrm{s}$, or roughly $2 \mathrm{BL} / \mathrm{s}$ (body lengths per second).

Trot gait speed The base trot frequency applied ( $f=$ $1.59 \mathrm{~Hz}$ ) resulted in an average robot speed of $v=$ $0.26 \mathrm{~m} / \mathrm{s}$, or $1.2 \mathrm{BL} / \mathrm{s}$. The fastest trot gait frequency was found at $3.98 \mathrm{~Hz}$, with $v=0.59 \mathrm{~m} / \mathrm{s}$, or $2.8 \mathrm{BL} / \mathrm{s}$. Speed of the robot increased mostly steadily until the maximum speed. The robot speed decreased for any higher control frequency, down to $v=0.41 \mathrm{~m} / \mathrm{s}$ at $f=$ $5.58 \mathrm{~Hz}$.

Pitch and roll angle phase plots Walk and trot gaits could be identified by their corresponding phase plots (e.g. Figure 16b, orange and blue), which changed with the applied locomotion frequency. Typically, walking patterns presented themselves with a higher roll than pitch angle range, compared to those of the corresponding trot gait patterns. This was likely due to the higher symmetry in trot footfall patterns, compared to walk footfall patterns. With increasing robot speed, roll and pitch angles range decreased (Figure 12). As only the system's frequency was increased, and no other parameter was altered, it is assumed that this stabilizing effect is mechanical. 


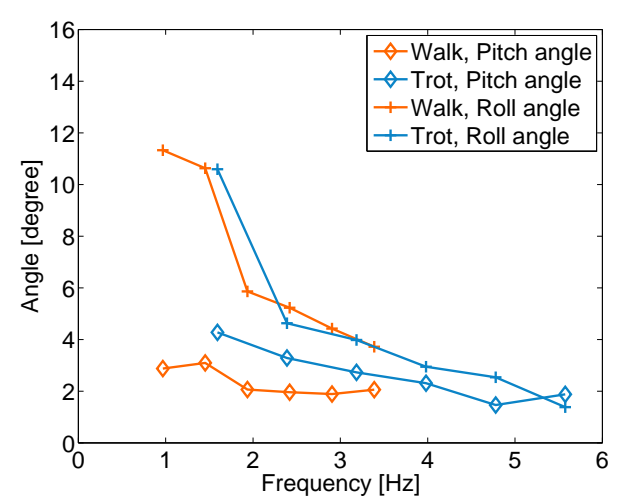

Fig. 12 Decreasing roll and pitch angle ranges, for increasing robot speed. All combinations for walk and trot, and roll and pitch are shown. Decreasing roll and pitch angles with increasing gait frequency indicate self-stabilizing properties of the applied gait.

\section{Transitions between walking and trotting}

The walk and trot gaits reconstructed from horse kMPs were the base gaits for the gait transition experiments conducted on the robot and reported in this section. Both the transition from walking to trotting and the transition from trotting to walking were generated according to the method described in Section 5.1. Trajectories obtained were applied on the compliant quadruped robot, and the results are documented in (Section 5.2).

\subsection{Methodology}

The foot trajectories of the four legs (Right Forelimb - RF, Left Forelimb - LF, Right Hindlimb - RH, Left Hindlimb - LH) for the walk and the trot gaits, derived as described in Section 4.1, are resampled to obtain 100 points per gait cycle. For each of the two gaits a "pivot" for the transition is identified using Equation (6):

$$
\begin{aligned}
{[\widehat{i}, \widehat{j}]=\min _{i, j} } & \left(d\left(R F_{\text {Walk }}(i), R F_{\text {Trot }}(j)\right)+\right. \\
+ & d\left(L F_{\text {Walk }}(i), L F_{\text {Trot }}(j)\right)+ \\
+ & d\left(R H_{\text {Walk }}(i), R H_{\text {Trot }}(j)\right)+ \\
+ & \left.d\left(L H_{\text {Walk }}(i), L H_{\text {Trot }}(j)\right)\right)
\end{aligned}
$$

Pivots indicate those points in the walk and the trot gaits, respectively, such that the cumulative distance (among the four legs) between walk trajectory and trot trajectory is minimal. In Figure 13, foot trajectories for walking and trotting are plotted together, and the pivot points of each gait are indicated with a dot. The transition period was set to take $3 / 4$ of a gait cycle to be completed: $3 / 8$ of trajectory before the pivot point,
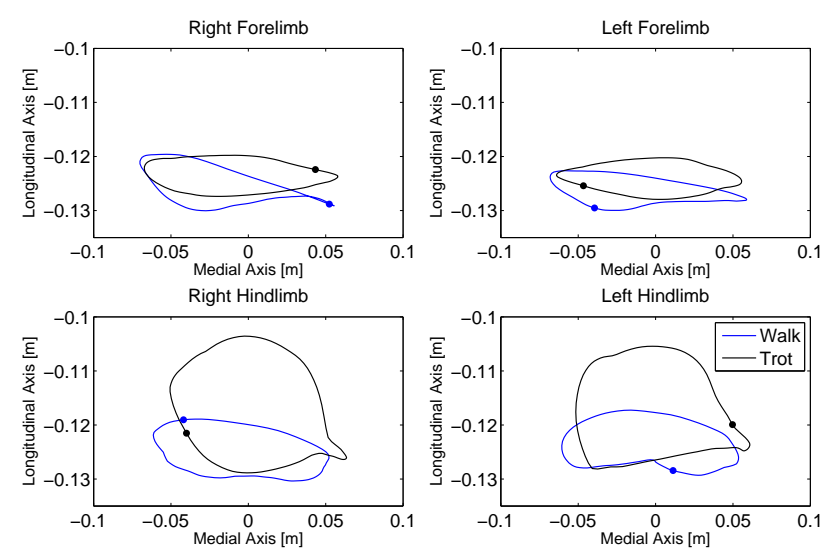

Fig. 13 The foot trajectories of walking and trotting. The dot indicates the pivot for the walk/trot transition.
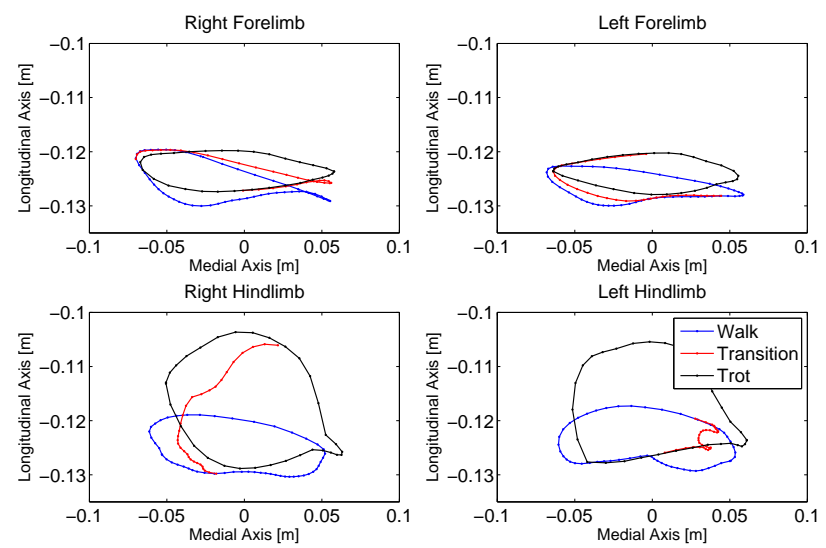

Fig. 14 The foot trajectories of walk, transition from walking to trotting, and trot
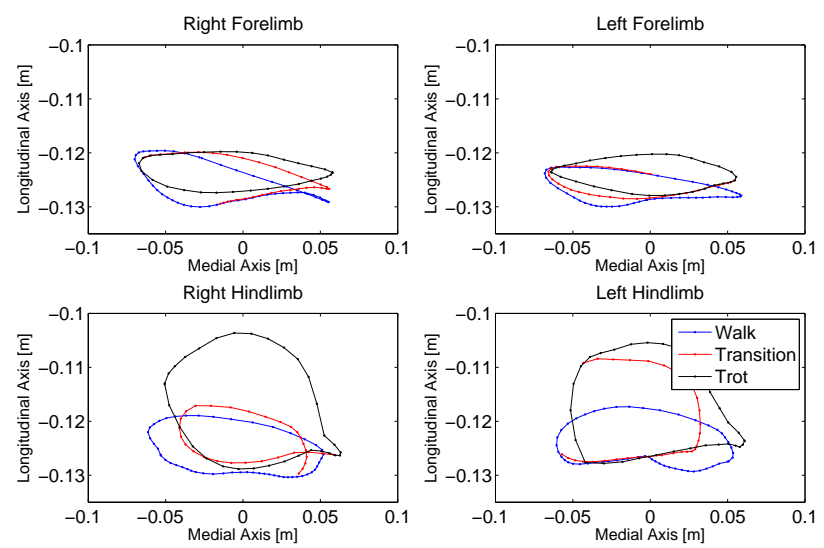

Fig. 15 The foot trajectories of trot, transition from trotting to walking, and walk

and $3 / 8$ of trajectory after the pivot point of each gait are considered. The walk to trot transition trajectory is a weighted average of the walk trajectory and the trot trajectory, with the weight of trot growing linearly from 0 to 1 , and the weight of walk decreasing linearly from 1 to 0 . 


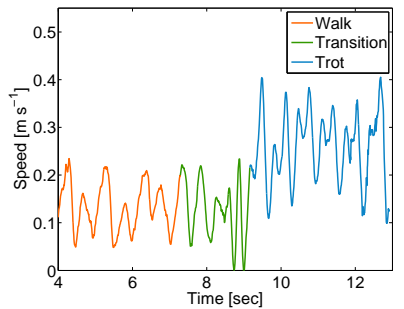

(a) $m=1$, w-t, speed

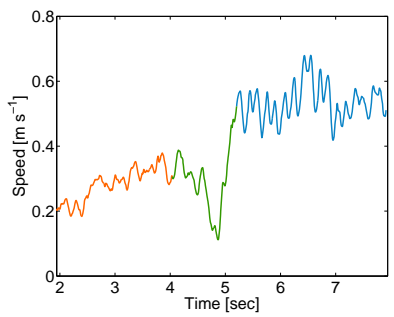

(e) $m=2$, w-t, speed

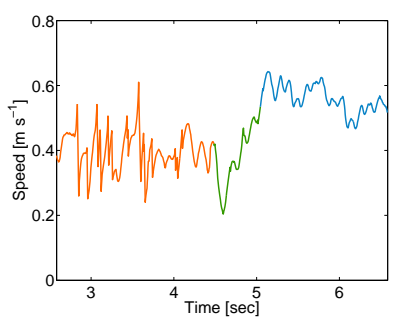

(i) $m=3$, w-t, speed

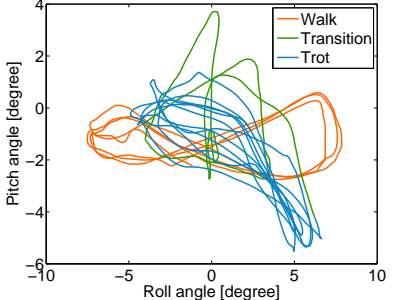

(b) $m=1, \mathrm{w}-\mathrm{t}$, phase plot

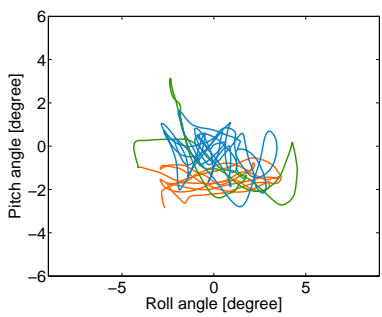

(f) $m=2$, w-t, phase plot

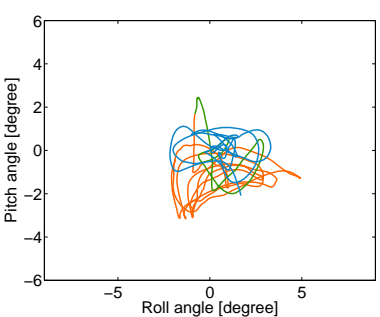

(j) $m=3$, w-t, phase plot

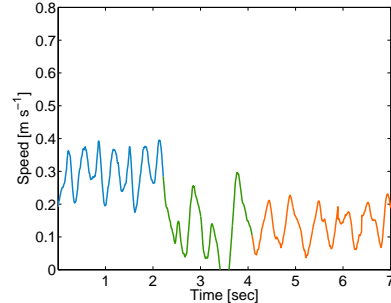

(c) $m=1, \mathrm{t}-\mathrm{w}$, speed

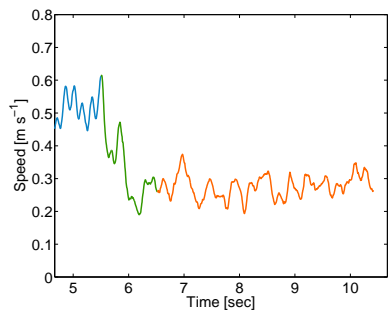

(g) $m=2, \mathrm{t}-\mathrm{w}$, speed

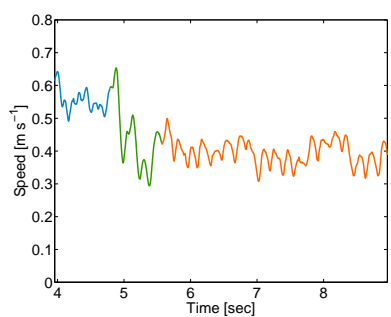

(k) $m=3, \mathrm{t}-\mathrm{w}$, speed

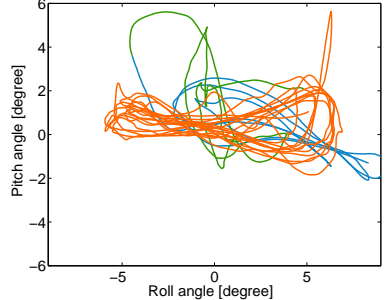

(d) $m=1$, t-w, phase plot

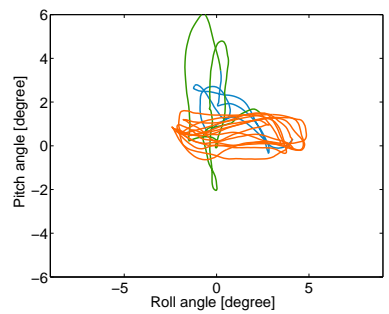

(h) $m=2$, t-w, phase plot

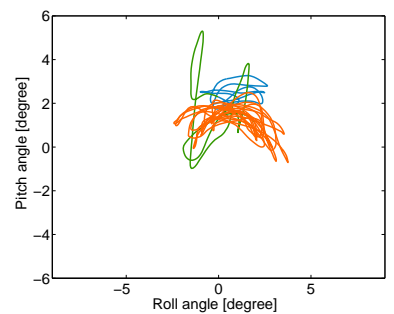

(l) $m=3, \mathrm{t}-\mathrm{w}$, phase plot

Fig. 16 Robot gait transition experiments with the three-quarter strategy (Table 5, plots of forward speed versus time and phase plots of roll and pitch angles. Rows from top to down: frequency multiplier $m=[1: 1: 3]$. Left two columns: walk-trot transition (w-t), right two columns: trot-walk transition ( $\mathrm{t}-\mathrm{w})$. For each run, instantaneous robot speed and roll-pitch data are plotted. Data was recored with the help of a motion capture system, which tracked three markers at the robot's trunk at $f=240 \mathrm{~Hz}$. Orange trajectories refer to walking gait patterns, green trajectories to gait transition, and blue trajectories to trot-gait patterns. Visible is a general decrease of pitching and rolling angles at increased robot speed. For low speed gait transition, instantaneous robot speed briefly drops to zero, less than $200 \mathrm{~ms}$, before it recovers. At higher speed, gait transition caused less prominent speed drops. In ideal cases (e.g. Fig. 16g and Fig. 16k) no speed drop is visible, gaits were switched with no speed perturbation to the system.

Walk and trot also have a different gait frequency that results in a different sampling rate for the respective trajectories. The transition trajectory, hence, has a variable sampling rate: in the case of the walk to trot transition this rate changes linearly from the sampling rate of the walk to that of the trot. Figure 14 shows the four foot trajectories during three different locomotion phases: a complete walk gait cycle, the transition from walk to trot, and a complete trot cycle. The samples are also shown. The reference motor positions derived from these data through inverse kinematics are sent to the robot at $f=50 \mathrm{~Hz}$.

The foot trajectories for the trot to walk transition are defined in a similar manner, just with the weight of the walk increasing linearly from 0 to 1 and the one of trot decreasing linearly from 1 to 0 , and the sampling rate going linearly from the sampling rate of trot to the sampling rate of walk, i.e., inverting the roles of walk and trot (Figure 15).

Both types, walk/trot and trot/walk transitions were tested on the compliant quadruped robot. Experiments on scaling the gait in frequency were held as well (scaling factor $m=[1: 0.5: 3.5])$. The results of these experiments are reported in the next section.

\subsection{Results robot gait transition}

The gait transition characteristics of the robot are analyzed by measuring the range of pitch and roll movements for each gait after reaching steady state, and during the transition phase. In addition, instantaneous speed was recorded before, during, and after each transition. Roll and pitch angle phase plots are shown in 
Table 5 Average transition times (in sec) for three transition strategies: Our method, No transition, and Not optimal pivot points. Average was taken over transition times of all multipliers. In average, our proposed method showed shortest transition times. Trot-walk transitions of the no-transition strategy had a tendency to produce non-stable walking gait patterns, with increasing pitch and roll angles (transition times only shown for stable runs).

\begin{tabular}{lrr} 
Strategy & Walk-Trot & Trot-Walk \\
\hline Our method & 1.2 & 1.3 \\
No transition & 1.7 & 1.5 \\
Not optimal pivot points & 1.4 & 1.3
\end{tabular}

Figure 16. Phase plots in Figure 16 are colored according to the commanded gait patterns, blue for trot gaits, orange for walking gait patterns, whilst green lines indicate transition times. The identical color coding was chosen for speed plots. Gait transitions were identified and marked manually by placing start and end with the help of the speed plots, as a first rough approximation. We then used phase plots and characteristic shapes of walk and trot phase patterns to more precisely separate transition times from steady gait locomotion. Qualitative plots are shown in Figure 16.

Frequency multiplication resulted typically in an increase in robot speed from walk to trot. All walk-trot and trot-walk gait transitions on flat ground were run successfully: the robot never stumbled nor fell. However, not all gait transitions worked equally well. One can see this by observing transition times and instantaneous transition robot speed (Figure 16). Typically, transitions went more smoothly with higher robot speed. In those cases transition happened more swiftly, and with less speed drop. Independently from the "direction" of the gait transition, stable patterns typically emerged after a transition time of roughly $t=1.2 / 1.3 \mathrm{~s}$.

A supplemental analysis was then performed to verify the effectiveness of our proposed method. The performance of the transitions (for both walk to trot and trot to walk, with frequency scaling factor from 1 to 3.5) as described in Section 5.1 in terms of transition time was compared to i) the "no transition" case, with a sudden switch from one gait to the other, and to ii) the case with a transition that happens around pivot points that are not optimal. For the latter we set as transition pivots those points in the walk and trot gaits such that the cumulative distance among the four legs between walk trajectory and trot trajectory is maximal, i.e., in this case Equation (6) is modified substituting min with $\max$.

The gait transition took $26 \%$ longer for the "no transition" strategy, compared to our proposed transition method. In the second case (non-optimal pivot points), the transition time was $9 \%$ longer. This indicates that the choice of good pivot points improves the quality of the gait transition (Table 5).

\section{Conclusions}

Horse kinematic Motion Primitives (kMPs) were extracted by applying PCA to the joint trajectories derived from motion capture data of horse locomotion. A comparative analysis, whose results are reported in Section 2, showed that a set of four kMPs, that together account for about $97 \%$ of variance, underlie the different horse gaits considered: walking, trotting, and galloping. This suggests that horses are capable of generating a vast variety of complex motions that are in fact based on a low dimensionality set of open-loop control signals, hence reducing the complexity of the coordination and control of the four limbs during locomotion.

The kMPs extracted were then used to generate horselike trajectories that were tested on a small quadruped. Using these gait profiles it was shown that the robot could successfully walk, trot, and gallop. The walk and trot gaits generated by reconstruction from kMPs were scaled in frequency (up to factor 3.5) to account for the smaller size of the robot compared to a horse, and the results of walking/trotting at higher frequencies were collected. In particular, the performance of the robot in terms of Cartesian velocity was evaluated in the different cases.

Finally, a gait transition strategy was proposed. The walk to trot transition and the trot to walk transition were successfully tested on the quadruped robot.

The main aims of the research presented in this paper were:

1. Get a better understanding of horse locomotion. The extraction of the kinematic Motion Primitives (kMPs) and the considerations on their nature are the results of this analysis.

2. Apply what has been learned from the analysis of horse locomotion to synthesize horse-like trajectories for quadruped robots. Walk, trot, and gallop gaits were generated and tested, and a transition strategy was proposed and tested, as well.

This research can be classified with the works in the literature that take inspiration from biology to generate motion for robots. Primitives are a possible solution. Another method that is widely adopted, and that is related to Primitives, is to use CPG. Primitives can be considered as the effect of CPG, observed at the level of muscles (Motor Primitives (MPs) extracted from EMG signals), or at the level of the kinematics (kinematic Motion Primitives (kMPs) extracted from joint angle 
trajectories). The method proposed in this paper uses kMPs to directly transfer the biological features of horse locomotion to the robot, that could successfully perform the gaits tested.

As a possible extension to this work, other transition criteria will be verified, and their performance will be compared to the results achieved with the method proposed in this paper. New tests of gallop will be performed, as well, with an increased friction between the feet and the ground to try to reduce the slippage observed when the robot is reproducing fast motions.

The nature of matrix $\mathrm{S}$ (Equation 1) will be further investigated, in order to allow a direct joint trajectories generation from kMPs.

\section{Acknowledgements}

This work is supported by the European Commission FP7, AMARSI Project ICT-2009-4.

\section{References}

Berns K, Ilg W, Deck M, Dillmann R (1998) The mammalian-like quadrupedal walking machine BISAM. In: International Workshop on Advanced Motion Control, Coimbra, Portugal

Billard A, Ijspeert AJ (2000) Biologically inspired neural controllers for motor control in a quadruped robot. In: International Joint Conference on Neural Networks, Come, Italy

Buehler M, Playter R, Raibert M (2005) Robots step outside. In: International Symposium on Adaptive Motion of Animals and Machines, Ilmenau, Germany

Canderle J, Caldwell DG (2000) A biologically inspired quadruped using pneumatic muscle actuators. In: International Conference on Climbing and Walking Robots, Madrid, Spain

Cappelletto J, Estavez P, Modina W, Fermin L, Bogado JM, Grieco JC, Fernando-Lopez G (2006) Gait synthesis and modulation for quadruped robot locomotion using a simple feed-forward network. In: International Conference on Artificial Intelligence and Soft Computing, Zakopane, Poland

Cappelletto J, Estevez P, Fernando-Lopez G, Grieco JC (2007) A cpg with force feedback for a statically stable quadruped gait. In: International Conference on Climbing and Walking Robots, Singapore

Chae KG, Park JH (2008) Trajectory optimization with ga and control for quadruped robots. Journal of Mechanical Science and Technology 23:114-123
Collins JJ, Richmond SA (1994) Hard-wired central pattern generators for quadrupedal locomotion. Biological Cybernetics 71:375-385

Degallier S, Righetti L, Natale L, Nori F, Metta G, Ijspeert A (2008) A modular bio-inspired architecture for movement generation for the infant-like robot iCub. In: 2nd IEEE RAS \& EMBS International Conference on Biomedical Robotics and Biomechatronics, Scottsdale, AZ, USA

Dégallier Rochat S, Ijspeert A (2010) Modeling discrete and rhythmic movements through motor primitives: a review. Biological Cybernetics 103(4):319-338

Fujii A, Saito N, Nakahira K, Ishiguro A, Eggenberger P (2002) Generation of an adaptive controller cpg for a quadruped robot with neuromodulation mechanism. In: IEEE International Workshop on Intelligent Robots and Systems, Lausanne, Switzerland

Fujita M, Kitano H (1998) Development of an autonomous quadruped robot for robot entertainment. Autonomous Robots 5(1):7-18

Fukuoka Y, Nakamura H, Kimura H (1999) Biologically-inspired adaptive dynamic walking of the quadruped on irregular terrain. In: International Conference on Control Applications, Kohala Coast, HI, USA

Fukuoka Y, Kimura H, Cohen AH (2003) Adaptive dynamic walking of a quadruped robot on irregular terrain based on biological concepts. International Journal of Robotics Research 22(2-3):187-202

Golubovic D, Hu H (2003a) Ga-based gait generation of sony quadruped robots. In: IASTED International Conference on Artificial Intelligence and Applications, Benalmadena, Spain

Golubovic D, Hu H (2003b) Parameter optimisation of an evolutionary algorithm for on-line gait generation of quadruped robots. In: IEEE International Conference on Industrial Technology

Griffin TM, Kram R, Wickler SJ, Hoyt DF (2004) Biomechanical and energetic determinants of the walk trot transition in horses. The Journal of Experimental Biology 207:4215-4223

Hebbel M, Nistico W, Fisseler D (2007) Learning in a high dimensional space: Fast omnidirectional quadrupedal locomotion. In: Lakemeyer G, Sklar E, Sorrenti D, Takahashi T (eds) RoboCup 2006: Robot Soccer World Cup X, Lecture Notes in Computer Science, vol 4434, Springer Berlin / Heidelberg, pp 314 321

Heglund NC, Taylor CR (1988) Speed, stride frequency and energy cost per stride: how do they change with body size and gait? The Journal of Experimental Biology 138:301-318 
Hirose S, Fukuda Y, Yoneda K, Nagakubo A, Tsukagoshi H, Arikawa K, Endo G, Doi T, Hodoshima R (2009) Quadruped walking robots at tokio institute of technology. IEEE Robotics \& Automation Magazine 16(2):104-114

Hoyt D, Taylor R (1981) Gait and the energetics of locomotion in horses. Nature 292:239240

$\mathrm{Hu} \mathrm{H}, \mathrm{Gu} \mathrm{D}$ (2005) Hybrid learning architecture for fuzzy control of quadruped walking robots. International Journal of Intelligent Systems - Soft Computing for Modeling, Simulation, and Control of Nonlinear Dynamical Systems 20(2):131-152

Iida F, Gomez GJ, Pfeifer R (2005) Exploiting body dynamics for controlling a running quadruped robot. In: IEEE International Conference on Robotics and Automation, Seattle, USA

Ijspeert AJ (2008) Central pattern generators for locomotion control in animals and robots: a review. Neural Networks 21(4)

Inagaki K, Kobayashi H (1993) A gait transition for quadruped walking machine. In: IEEE/RSJ International Conference on Intelligent Robots and Systems, Yokohama, Japan

Ishii T, Masakado S, Ishii K (2004) Locomotion of a quadruped robot using cpg. In: IEEE International Joint Conference on Neural Networks, Budapest, Hungary

Kim HK, Ohung W, Won D, Park S, Kim TJ, Kim SS (2008) Foot trajectory generation of hydraulic quadruped robots on uneven terrain. In: IFAC World Congress, Seoul, Republic of Korea

Kim KY, OKwon, Yeon JS, Park JH (2006) Elliptic trajectory generation for galloping quadruped robots. In: IEEE International Conference on Robotics and Biomimetics, Kunming, China

Kimura H, Shimoyama I, Miura H (1989) Dynamics in the dynamic walk of a quadruped robot. Advanced Robotics 4(3):283-301

Kimura H, Fukuoka Y, Cohen AH (2007) Adaptive dynamic walking of a quadruped robot on natural ground based on biological concepts. International Journal of Robotics Research 26(5):475-490

Kramy DP, Orin DE (2003) Achieving periodic leg trajectories to evolve a quadruped gallop. In: IEEE International Conference on Robotics and Automation, Tapei, Taiwan

Lin JN, Song SM (2002) Modeling gait transitions of quadrupeds and their generalization with cmac neural networks. In: IEEE International Conference on Systems, Man and Cybernetics, Yasmine Hammamet, Tunisia

Liu C, Chen Y, Zhang J, Chen Q (2009) Cpg driven locomotion control of quadruped robot. In: IEEE In- ternational Conference on Systems, Man and Cybernetics, San Antonio, Texas, USA

Moro FL, Tsagarakis NG, Caldwell DG (2011) A human-like walking for the COmpliant huMANoid COMAN based on CoM trajectory reconstruction from kinematic Motion Primitives. In: 11th IEEERAS International Conference on Humanoid Robots, Bled, Slovenia, pp 364-370

Moro FL, Tsagarakis NG, Caldwell DG (2012a) Efficient human-like walking for the COmpliant huMANoid COMAN based on kinematic Motion Primitives (kMPs). In: IEEE International Conference on Robotics and Automation, Saint Paul, MN, USA

Moro FL, Tsagarakis NG, Caldwell DG (2012b) On the Kinematic Motion Primitives (kMPs): Theory and Application. Frontiers in Neurorobotics 6(10):1-18

Nichol JG, Singh SPN, Waldron KJ, Palmer III LR, Orin DE (2004) System design of a quadrupedal galloping machine. International Journal of Robotics Research 23(10-11):1013-1027

Pongas D, Mistry M, Schaal S (2007) A robust quadruped walking gait for traversing rough terrain. In: IEEE International Conference on Robotics and Automation, Roma, Italy

Poulakakis I, Smith JA, Buehler M (2005) Modeling and experiments of untethered quadrupedal running with a bounding gait: the scout ii robot. International Journal of Robotics Research 24(4):239256

Raibert M (1986) Legged robots that balance. MIT Press

Raibert M, Blankespoor K, Nelson G, Playter R, and the BigDog Team (2008) Bigdog, the roughterrain quadruped robot. In: IFAC World Congress, Seoul, Republic of Korea

Raibert MH (1990) Trotting, pacing and bounding by a quadruped robot. Journal of Biomechanics 23(1):8398

Rebula JR, Neuhaus PD, Bonnlander BV, Johnson MJ, Pratt JE (2007) A controller for the littledog quadruped walking on rough terrain. In: IEEE International Conference on Robotics and Automation, Roma, Italy

Righetti L, Ijspeert AJ (2008) Pattern generators with sensory feedback for the control of quadruped locomotion. In: IEEE International Conference on Robotics and Automation, Pasadena, CA, USA

Rutishauser S, Sproewitz A, Righetti L, Ijspeert AJ (2008) Passive compliant quadruped robot using central pattern generators for locomotion control. In: IEEE International Conference on Biomedical Robotics and Biomechatronics, Scottsdale, AZ, USA Sakakibara Y, Kan K, Hosoda Y, Hattori M, Fujie M (1990) Foot trajectory for a quadruped walking ma- 
chine. In: IEEE International Workshop on Intelligent Robots and Systems, Ibaraki, Japan

Semini C, Tsagarakis NG, Guglielmino E, Focchi M, Cannella F, Caldwell DG (2011) Design of hyq a hydraulically and electrically actuated quadruped robot. Proceedings of the Institution of Mechanical Engineers, Part I: Journal of Systems and Control Engineering 225(1):831-849

Son Y, Kamano T, Yasuno T, Suzuki T, Harada $\mathrm{H}$ (2006) Generation of adaptive gait patterns for quadruped robot with cpg network including motor dynamic model. Electrical Engineering in Japan 155(1):35-43

Spröwitz A, Tuleu A, Vespignani M, Ajallooeian M, Badri E, Ijspeert AJ (2012) Towards dynamic trot gait locomotion-design, control, and experiments with cheetah-cub, a compliant quadruped robot (under review)

Tsujita K, Toui H, Tsuchiya K (2005) Dynamic turning control of a quadruped locomotion robot using oscillators. Advanced Robotics 19(10):1115-1133

Vilensky JA, Libii JN, Moore AM (1991) Trot-gallop gait transitions in quadrupeds. Physiology \& Behavior 50(4):835-842

Witte H, Hackert R, Ilg W, Biltzinger J, Schillinger N, Biedermann F, Jergas M, Preuschoft H, Fischer MS (2003) Quadrupedal mammals as paragons for walking machines. In: Proc AMAM - Adaptive Motion in Animals and Machines

Zhang X, Zheng H, Guan X, Chifeng Z, Zhao L (2005) A biological inspired quadruped robot: structure and control. In: IEEE International Conference on Robotics and Biomimetics, Hong Kong, China 